Chapter 24

\title{
Plasma Process on Ionic Liquid Substrate for Morphology Controlled Nanoparticles
}

\author{
Toshiro Kaneko, Shohei Takahashi and \\ Rikizo Hatakeyama \\ Additional information is available at the end of the chapter \\ http://dx.doi.org/10.5772/52095
}

\section{Introduction}

The interaction of discharge plasmas with liquids [1-5] is one of the active topics in the realm of recent plasma science and science technology. It has pioneered new channels relating to nano material creation based on their distinct properties such as ultra-high density, high reactivity, high process rate, and so on. Especially, the boundary between the plasmas and the liquids, which activates physical processes and chemical reactions, has attracted much attention as a novel field in the nano-bio material creation. For example, the nanoparticle synthesis using the plasma-liquid interfaces [6-10] is especially advantageous in that a reducing agent is the plasma itself, and then, toxic stabilizers and reducing agents are unnecessary and the synthesis is continuous during the plasma irradiation. In these methods, although it has been reported that the metal salt is reduced by an electron or an active hydrogen, the precise control of the synthesis in terms of the synthesis rate, morphology (size, shape, structure, and so on) control remain unclear because the inevitable high voltage discharge in the atmospheric pressure and the consequential dynamic behavior of the gas-liquid interface prevent us from analyzing the precise properties of the plasmas in the interfacial region.

In this sense, for the purpose of the generation of the static and stable plasma contacting with the liquid, we adopt ionic liquids [11,12] which have the interesting characteristics such as their composition consisting of only positive and negative ions, i.e., no neutral solvent, extremely low vapor pressure, high heat capacity, and nonflammability. These characteristics enable us to introduce the ionic liquids to the vacuum system and the discharge plasma. Therefore, the ionic liquids are the most suitable liquid for the formation of nano-composite materials using the discharge plasmas in contact with the liquids [13-21]. 
On the other hand, recently, highly-ordered periodic structures of metal nanoparticles have attracted much attention due to their high catalytic activity, unique photosensitive reactivity, bio sensitivity, and so on [22-26]. One possibility is use of nano-carbons such as carbon nanotubes or graphenes as template for synthesis of the nanoparticles [27-32]. However, the structures of the nanoparticles are decided by the chemical properties of the nano-carbons and are difficult to be freely controlled by the external parameters. To realize the easy and flexible control of the periodic structure of the nanoparticles, we adopt a novel plasma technique combined with introduction of ionic liquids under strong magnetic fields up to several tesla (T), whose concept is schematically shown in Figure 1 [33].

Since the plasma generated under the strong magnetic field keeps its structure due to confinement along the magnetic field lines, the plasma structure can be transcribed to the liquid surface, resulting in the synthesis of the structured nanoparticles at the gas-liquid interface when the plasma reduces the metal chlorides in the liquid. This method could contribute to supplying a considerable amount of spatially-periodic nanoparticles available for the development of unique optoelectronic devices [34].

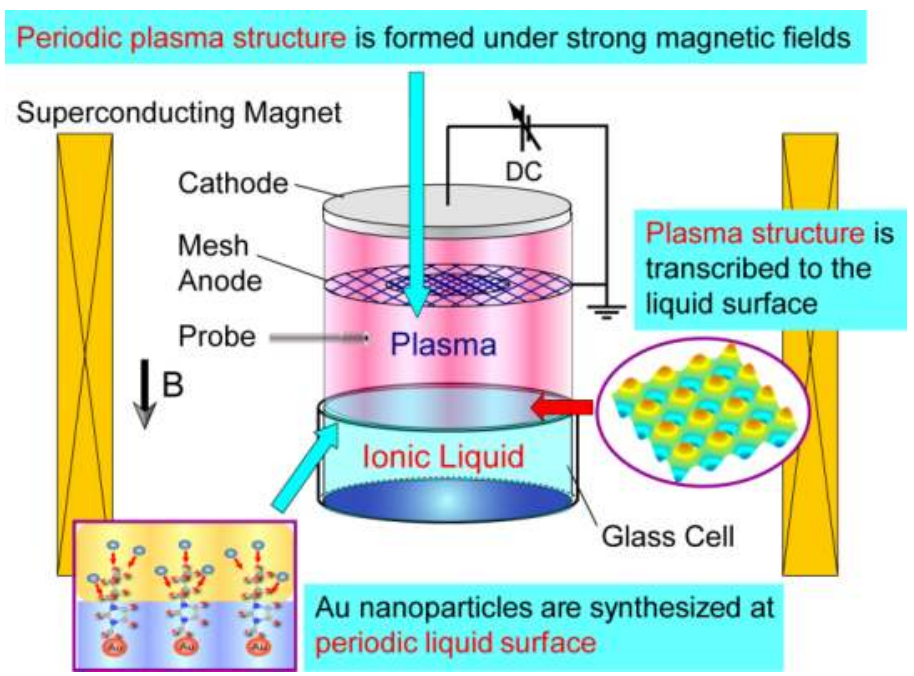

Figure 1. Concept of synthesis of periodic metal nanoparticles using discharge plasmas in contact with ionic liquid substrate.

\section{Gas-liquid interfacial plasma process using ionic liquid substrate}

Figure 2 shows schematic model of the gas-liquid interfacial plasmas for Au nanoparticle synthesis. An electrode which is made of a platinum (Pt) plate is located inside the glass cell, and a popular ionic liquid (1-buthl-3-methyl-imidazolium tetrafluoroborate: $\left[\mathrm{C}_{8} \mathrm{H}_{15} \mathrm{~N}_{2}\right]^{+}\left[\mathrm{BF}_{4}\right]^{-}$) is in- 
troduced on the Pt electrode as cathode electrodes for the purpose of investigating the effects of the ionic liquid on the discharge. On the other hand, a grounded anode electrode which is made of the SUS plate is set in a gas phase (plasma) region at a distance of $60 \mathrm{~mm}$ from the cathode electrodes. This discharge configuration, in which the ionic liquid cathode electrode is in the glass cell, is defined as "ion irradiation mode", because the positive ions in the plasma are accelerated by the electric field formed on the ionic liquid as shown in Figure 2(a).

In order to examine the effects of the ion irradiation to the ionic liquid on discharge-related phenomena, the cathode electrode is switched to the SUS plate located at the top of the gas plasma region, which is defined as "electron irradiation mode" and the anode electrode consisting of the ionic liquid in the glass cell is grounded as shown in Figure 2(b). Removal of the water dissolved in the ionic liquid is performed under the vacuum condition for 2 hours after introducing the ionic liquid into the glass chamber. A negative direct current (DC) voltage is supplied to the cathode electrode, where typical discharge voltage $V_{\mathrm{D}}$, discharge current $I_{\mathrm{D}}$, plasma irradiation time $t_{\mathrm{pi}}$ are $V_{\mathrm{D}}=500 \sim 1500 \mathrm{~V}, I_{\mathrm{D}}=1 \sim 5 \mathrm{~mA}, t_{\mathrm{pi}}=1 \sim 40 \mathrm{~min}$, respectively. The argon gas is adopted as a discharge medium, and the gas pressure $P_{\text {gas }}$ is varied from $20 \mathrm{~Pa}$ to $200 \mathrm{~Pa}$ approximately. A Langmuir probe is inserted at the position of $z$ $=0 \sim 60 \mathrm{~mm}$ to measure parameters of the plasma in contact with the ionic liquid substrate $(z$ $=0$ : surface of the ionic liquid substrate in the glass cell).

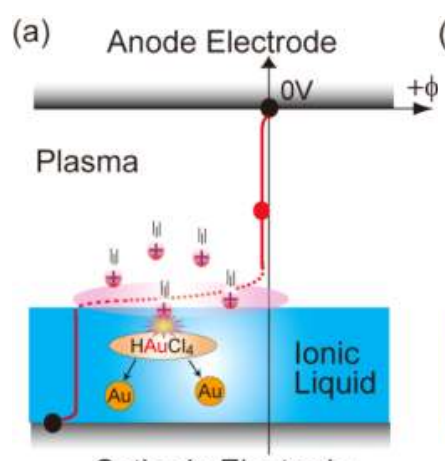

Cathode Electrode (b) Cathode Electrode

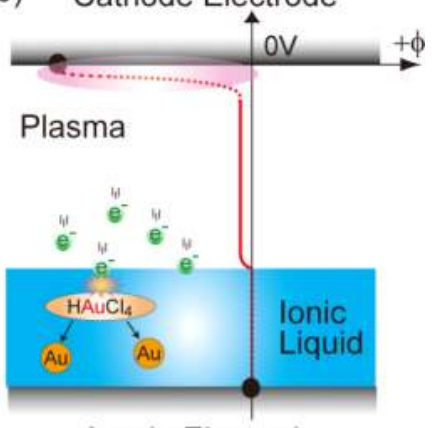

Anode Electrode

Figure 2. Schematic model of nanoparticle synthesis in the gas-liquid interface on the ionic liquid substrate. (a) ion irradiation mode, (b) electron irradiation mode.

Using this ion or electron irradiation, gold nanoparticles are synthesized in the ionic liquid by the reduction of $\mathrm{Au}$ chloride such as $\mathrm{HAuCl}_{4}$ dissolved in the ionic liquid. Figure 3 shows transmission electron microscopy (TEM) images of the Au nanoparticles synthesized in (a) the ion irradiation mode and (b) the electron irradiation mode for $P_{\text {gas }}=60 \mathrm{~Pa}, I_{\mathrm{D}}=1 \mathrm{~mA}$, and $t_{\mathrm{pi}}=40 \mathrm{~min}$. In both cases, the Au nanoparticles can be formed, however, it is found that, in ion irradiation mode, the average diameter of the Au nanoparticles is smaller and the particle number is larger than that in electron irradiation mode. The reduction reaction of the $\mathrm{Au}$ ions is believed to be caused by electrons injected from the plasma in electron irradiation 
mode, while in ion irradiation mode, the reduction may be caused by the hydrogen radical $\mathrm{H}^{*}$, which is generated by the dissociation of the ionic liquid. Based on this mechanism, the hydrogen radical is considered to be more effective for the reduction of Au ions than electrons, and efficient Au nanoparticle synthesis is realized using ion irradiation.

Since Au nanoparticles with diameter less than $100 \mathrm{~nm}$ are known to exhibit localized surface plasmon resonance, visible absorption spectra are obtained for a quantitative observation of the Au nanoparticle concentration. Figure 3(c) shows visible absorption spectra of the Au nanoparticles synthesized by an Ar plasma in ion and electron irradiation mode. The absorption peak appears around $550 \mathrm{~nm}$, corresponding to the Au plasmon resonance, and the absorption-peak intensity in ion irradiation mode is obviously larger than that in electron irradiation mode. Ar ions with high energy can penetrate deep into the ionic liquid, promoting the generation of hydrogen radicals. The increased concentration of hydrogen radicals may reduce Au ions more effectively in ion irradiation mode than electron irradiation mode. The rate of Au nanoparticle synthesis could be controlled by the irradiation energy of inert gas ions such as Ar.
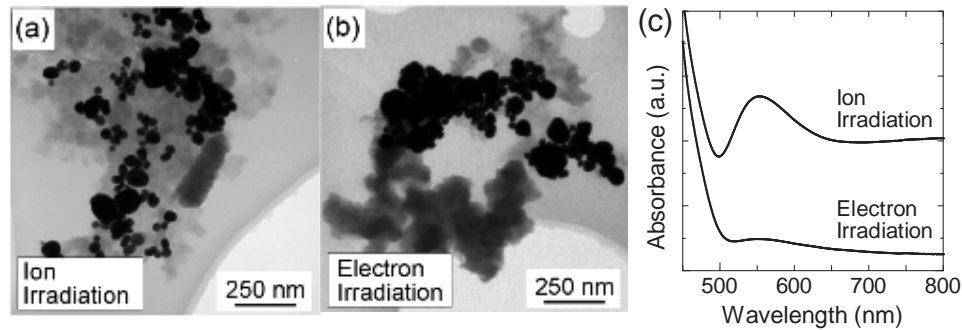

Figure 3. TEM images of Au nanoparticles synthesized in (a) the ion irradiation mode and (b) the electron irradiation mode. (c) UV-Vis absorption spectra of Au nanoparticles. $P_{\text {gas }}=60 \mathrm{~Pa}, I_{\mathrm{D}}=1 \mathrm{~mA}, t_{\mathrm{pi}}=40 \mathrm{~min}$.

\section{Control of nanoparticle morphology by gas-liquid interfacial plasmas}

\subsection{Periodic nanoparticle structure formed by periodic plasma}

Figure 4(a) shows the schematic of an experimental setup for nanoparticle structure formation using the gas-liquid interfacial discharge plasma under strong magnetic fields, which has a glass cell with $15 \mathrm{~mm}$ inner diameter and $10 \mathrm{~mm}$ depth in a cylindrical glass chamber with $75 \mathrm{~mm}$ diameter and $200 \mathrm{~mm}$ length. A DC voltage $V_{\mathrm{D}}$ is supplied to an upper cathode electrode composed of a stainless steel (SUS) plate and a SUS mesh grid (10 meshes/inch) is used as an anode electrode to promote a spatial diffusion of the plasma. Typical discharge current is $I_{\mathrm{D}}=3 \mathrm{~mA}$. Nitrogen gas is adopted as a discharge gas, and the gas pressure $P_{\text {gas }}$ is varied from 20 to $100 \mathrm{~Pa}$.

The new kind of the ionic liquid (N.N.N.-Trimethyl-N-propyl-ammonium Bis (trifluoro methane sulfonyl) imide) put in the glass cell is placed on a peltier element which is located at a distance of $50 \mathrm{~mm}$ from the anode electrode. Since this ionic liquid does not become supercooled 
state, we can make the ionic liquid solid state by cooling the ionic liquid using the peltier element located under the glass cell. When the strong magnetic fields are applied along the machine axis, the generated plasma is strongly magnetized, and then, the periodic plasma structure formed by the mesh anode is maintained just above the ionic liquid as shown in Figure 4(b).

The Au nanoparticles are synthesized in the ionic liquid by the plasma reduction of $\mathrm{HAuCl}_{4}$. The ionic liquid can be cooled by the peltier element and becomes the solid state as mentioned above, with keeping the structure of Au nanoparticles synthesized by the plasma irradiation at the liquid interface.
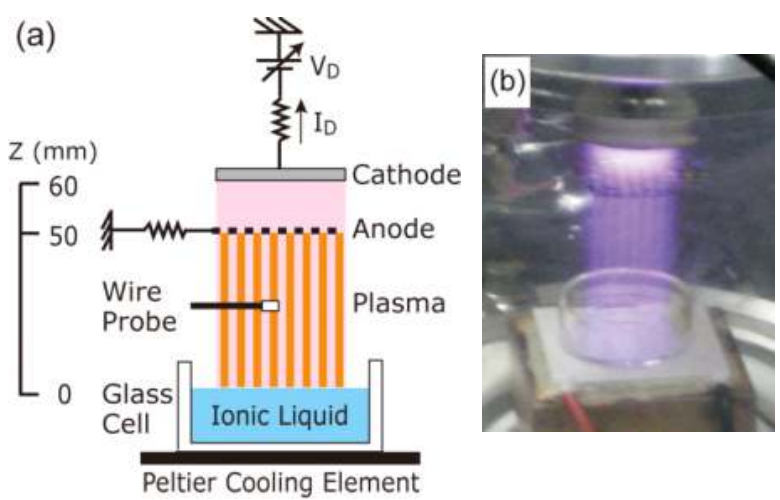

Figure 4. a) Schematic of the experimental setup and (b) photo of the synthesized periodic plasma structure for $B=1$ $\mathrm{T}, I_{\mathrm{D}}=3 \mathrm{~mA}, P_{\text {gas }}=20 \mathrm{~Pa}$.

Figure 5 shows the radial profiles of the electron density $n_{\mathrm{e}}$ of the plasma as functions of (a) magnetic fields $B$ and (b) gas pressure $P_{\text {gas }}$. When the strong mantic fields is applied (closed circles), the electron density has the depression periodically in the radial direction. The electron density in the high density region is about $10^{8} \mathrm{~cm}^{-3}$, and that in the depression region becomes one order smaller. The interval of the depression is about $2.5 \mathrm{~mm}$, which is corresponding to the distance between the SUS wire of the mesh anode electrode. Therefore, these density depressions are caused by shielding of the plasma under the wire of the mesh anode, where the generated plasma cannot pass through toward the ionic liquid substrate. In the absence of the magnetic field, on the other hand, the radial profile of the electron density is almost flat even using the mesh anode, because the plasma diffuses in the radial direction and becomes uniform.

When the gas pressure is changed from $P_{\text {gas }}=20 \mathrm{~Pa}$ to $40 \mathrm{~Pa}$, the radial density profile drastically changes as shown in Figure 5(b). Since the collision between the ions and neutral particles becomes frequent with an increase in the gas pressure, the formed periodic structure of the electron density collapses and becomes relatively flat.

Based on these results, it is found that the structure of the plasma is sensitive to the magnetic field and gas pressure, which are necessary to be carefully adjusted to obtain the desired plasma structure. 

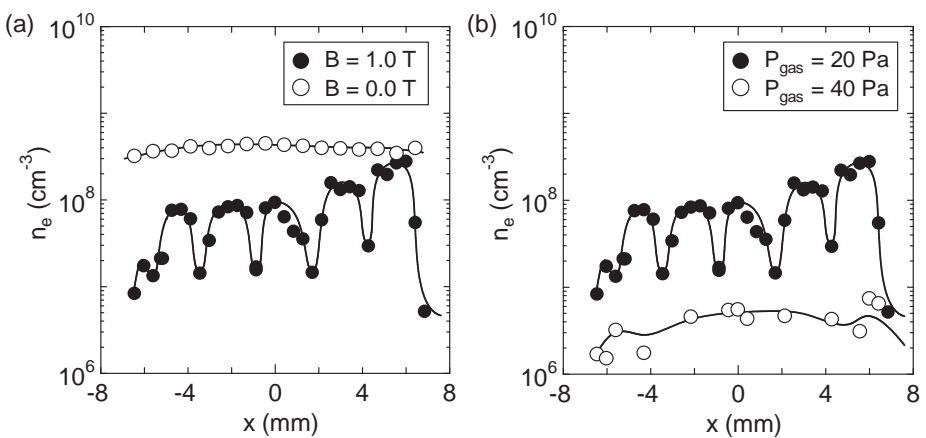

Figure 5. Radial profiles of the electron density of the plasmas (a) as a function of magnetic fields $B$ for $P_{\text {gas }}=20 \mathrm{~Pa}$ and (b) as a function of gas pressure $P_{\text {gas }}$ for $B=1 \mathrm{~T} . I_{\mathrm{D}}=3 \mathrm{~mA}$.

Using this periodic plasma structure, we attempt to synthesize the Au nanoparticles with periodic morphology. Figure 6 shows photos of the temporal evolution of the periodic $\mathrm{Au}$ nanoparticle structure which is formed at the gas-liquid interface in accordance with the periodic plasma structure under the condition of the strong magnetic field of $B=1 \mathrm{~T}$. The density of the periodic structured Au nanoparticles increases with plasma irradiation time $t_{\mathrm{pi}}$ and the obvious structure is formed typically within $t_{\mathrm{pi}}=5 \mathrm{~min}$. The interval of the nanoparticle structure is about $2.5 \mathrm{~mm}$, which is same as the distance between the wires of the mesh anode. As mentioned above, the electron density decreases under the shadow region of the mesh anode. Therefore, the nanoparticles are considered to be synthesized in the electron density depression region. This synthesis mechanism is discussed later.

In order to analyze the properties of the periodic structured Au nanoparticles, the ionic liquid is cooled using the peltier element located under the glass cell and is changed to the solid phase. The solid phase ionic liquid containing the Au nanoparticles can be extracted from the vacuum chamber with keeping its structure. Figure 7 shows the picture of the periodic structure of the $\mathrm{Au}$ nanoparticles which are synthesized on the ionic liquid substrate at room temperature, and (a) kept at room temperature and (b) cooled under $T_{\text {sub }} \sim 0{ }^{\circ} \mathrm{C}$ after the synthesis. The ionic liquid becomes solid state under $T_{\text {sub }}=0{ }^{\circ} \mathrm{C}$ and the Au nanoparticles are fixed in the solid state of the ionic liquid.

However, when the plasma is irradiated for longer than $5 \mathrm{~min}$, the Au nanoparticles diffuse and the structure is broadened as shown in Figure 7(b). In order to suppress the diffusion of the Au nanoparticles, the temperature of the ionic liquid is reduced during the plasma process using the peltier element, resulting in the increase in viscosity of the ionic liquid. Although the diffusion of the Au nanoparticles is suppressed, the Au nanoparticle synthesis rate becomes low. Therefore, it is necessary to precisely control the temperature of the ionic liquid for the fine periodic Au nanoparticle structure. 

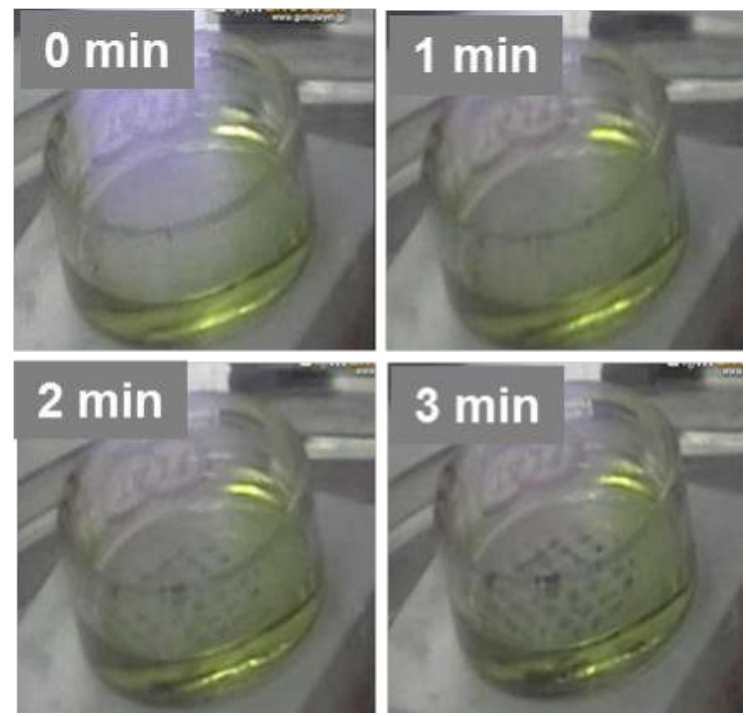

Figure 6. Temporal evolution of the periodic nanoparticle structure formed by controlled gas-liquid interfacial plasmas. $P_{\text {gas }}=20 \mathrm{~Pa}, I_{\mathrm{D}}=3 \mathrm{~mA}, B=1 \mathrm{~T}$.
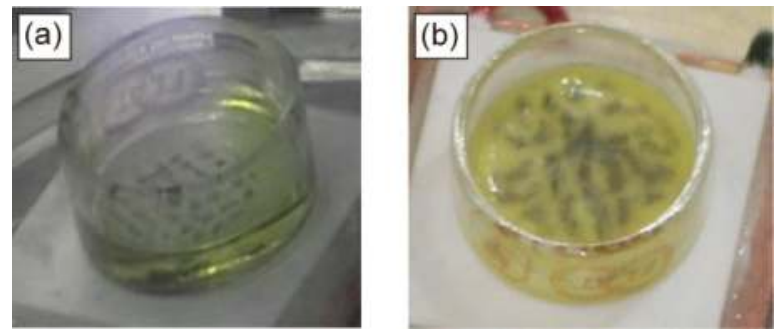

Figure 7. Pictures of the periodic nanoparticle structures which are formed at room temperature and (a) kept at room temperature and (b) cooled under $T_{\text {sub }}=0^{\circ} \mathrm{C} . P_{\text {gas }}=20 \mathrm{~Pa}, I_{\mathrm{D}}=3 \mathrm{~mA}, B=1 \mathrm{~T}$.

\subsection{Ring-shaped nanoparticle structure formed by structure-controlled plasma}

As the next step, we attempt to form more finely periodic structures of the Au nanoparticles based on the self-organizing behavior of turbulent plasmas generated by the nonlinear development of plasma fluctuations. For this purpose, a ring electrode is inserted in the plasma column as shown in Figure 8, and a positive DC bias voltage $V_{\mathrm{RG}}$ is applied to the electrode. It is found that the high frequency fluctuation $(100 \mathrm{kHz}-1 \mathrm{MHz})$ is excited by the positive bias voltage, however the self-organized plasma structure is not observed at present. Therefore, in this experiment, the ring electrode is used for the plasma structure control without bias voltage. 
Using this configuration, the Au nanoparticles are synthesized by reducing $\mathrm{HAuCl}_{4}$. It is found that the ring shaped Au nanoparticle structure is formed corresponding to the shape of the inserted ring electrode as shown in Figure 8(b). This result means that the Au nanoparticles are synthesized at the region without plasma irradiation due to the shielding by the ring electrode. Although the Au nanoparticles are usually synthesized by the reduction effect of the electrons in the plasma [14-16], the electron is absent in the shadow region of the ring electrode in this experiment. Therefore, the reducing agent is not electrons in this case.

(a)

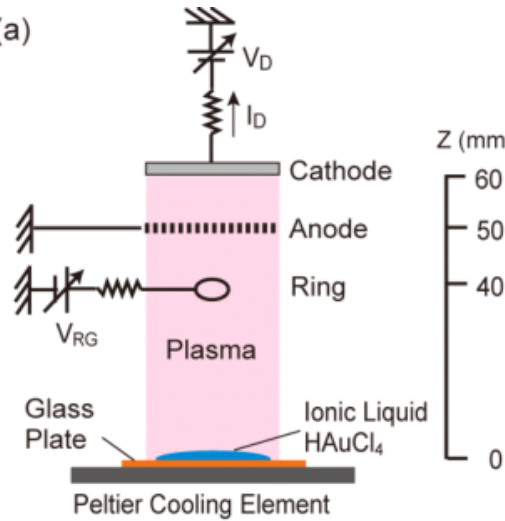

(b)

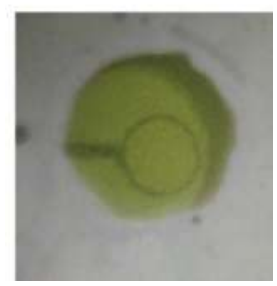

Figure 8. a) Experimental apparatus using ring electrode for nanoparticle structure control and (b) the synthesized ring shaped nanoparticle structure $B=1 \mathrm{~T}, I_{\mathrm{D}}=2 \mathrm{~mA}, P_{\text {gas }}=20 \mathrm{~Pa}$.

To explain the phenomena, we use the model of the Au nanoparticle synthesis as shown in Figure 9(a). As mentioned above, the charged particles such as the electrons and the positive ions cannot reach to the shadow region of the ring electrode, namely, only neutral radicals can arrive at the shadow region. The ionic liquid used in this experiment is described in Figure 9(b), which has $\mathrm{C}-\mathrm{H}$ bond in cation (positive ion) and C-F bond in anion (negative ion). When the radicals in the plasma are irradiated to the ionic liquid, the $\mathrm{C}-\mathrm{H}$ bond of the ionic liquid is considered to be dissociated, and the generated hydrogen radicals reduce the Au ions, resulting in the synthesis of Au nanoparticles in the shadow region of the ring electrode. On the other hand, in the plasma irradiation region, relatively high-energy ions are irradiated to the ionic liquid, and the $C-F$ bond whose dissociation energy $(D=5.07 \mathrm{eV})$ is larger than that of the $\mathrm{C}-\mathrm{H}$ bond $(\mathrm{D}=4.29$ $\mathrm{eV})$, can be dissociated by the high-energy ions. Therefore, the Au nanoparticles are destroyed by the oxidation effect of the fluorine radicals which come from the dissociation of the ionic liquid by the collision with the high energy charged particle.

To understand the mechanism of this ring-shaped nanoparticle structure formation, the ring electrode is changed to disk electrode as shown in Figure 10, where the bias voltage of the disk electrode $V_{\mathrm{DK}}$ is floated in this experiment. In this case, since the shadow region of the disk electrode is wide and clear compare with the ring electrode, it is possible to clarify the 
species in the plasma, which are necessary for the synthesis of the Au nanoparticles. To change the plasma characteristics irradiated to the ionic liquid, we change the discharge current $I_{\mathrm{D}}$.

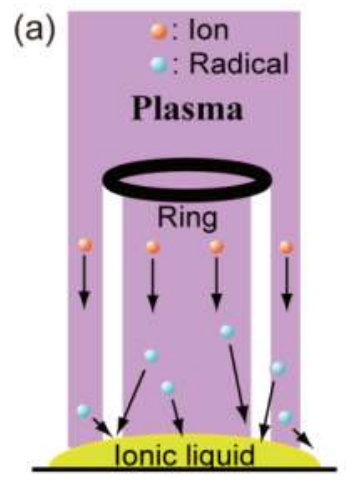

(b)

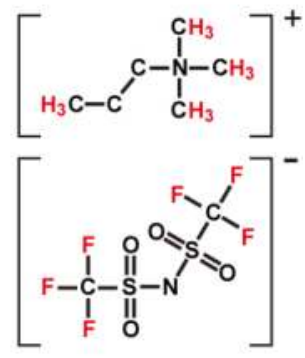

Figure 9. a) Model of the synthesis mechanism of the ring shaped Au nanoparticles and (b) chemical formula of the ionic liquid used in this experiment.

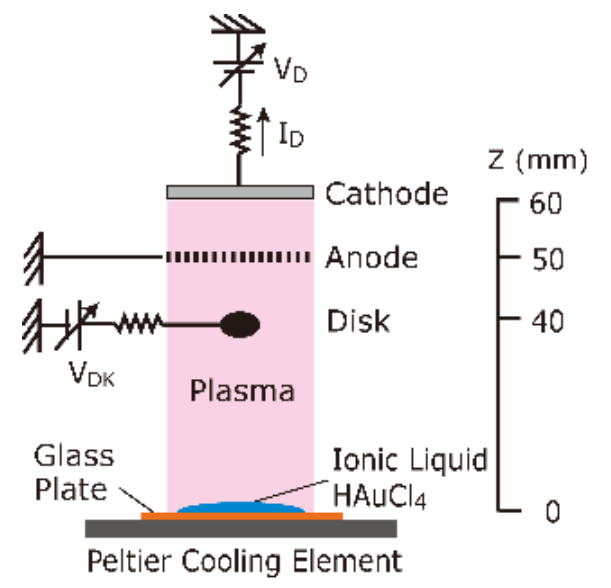

Figure 10. Experimental apparatus using disk electrode for understanding the mechanism of nanoparticle structure formation.

Figure 11 shows snapshots of the temporal evolution of the Au nanoparticle synthesis for (a) $I_{\mathrm{D}}=1 \mathrm{~mA}$ and (b) $I_{\mathrm{D}}=3 \mathrm{~mA}$. In the case for $I_{\mathrm{D}}=1 \mathrm{~mA}$, the Au nanoparticles are synthesized from the outside of the ionic liquid in the plasma irradiation region, and are absent in the shadow region of the disk electrode for the plasma irradiation time $t_{\mathrm{pi}}=5 \mathrm{~min}$. In the case of $I_{\mathrm{D}}=3 \mathrm{~mA}$, on the other hand, the Au nanoparticles are synthesized not only from the outside 
of the ionic liquid but also the edge region of the disk electrode for $t_{\mathrm{pi}}=40 \mathrm{sec}$. Furthermore, the Au nanoparticles are not synthesized in the plasma irradiation region.

(a)

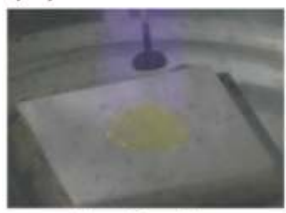

$\mathrm{tpi}=0 \mathrm{~min}$

(b)

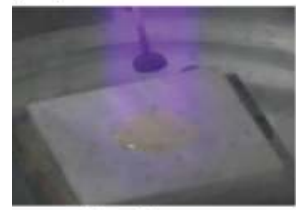

$\mathrm{tp}=0 \mathrm{sec}$

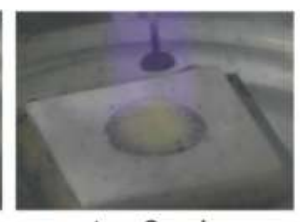

$t_{p i}=3 \mathrm{~min}$

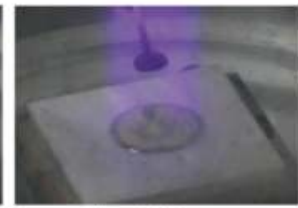

$t_{p i}=20 \mathrm{sec}$

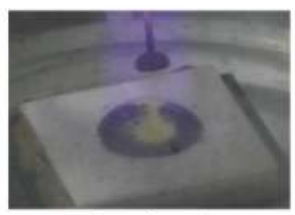

tpi $=4 \mathrm{~min}$

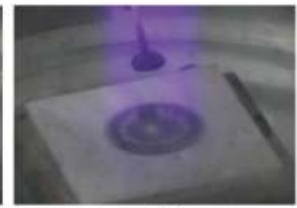

$\mathrm{tpi}=40 \mathrm{sec}$

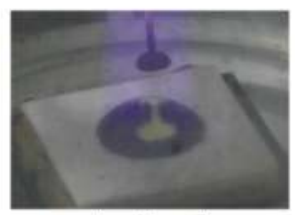

$t_{\mathrm{pi}}=5 \mathrm{~min}$

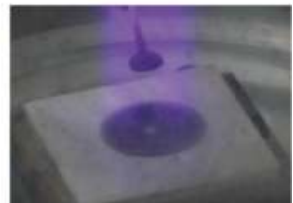

$t_{\mathrm{pi}}=60 \mathrm{sec}$

Figure 11. Photos of the Au nanoparticle structure as a function of plasma irradiation time $t_{\mathrm{pi}}$ for (a) $I_{\mathrm{D}}=1 \mathrm{~mA}$ and $(\mathrm{b})$ $I_{\mathrm{D}}=3 \mathrm{~mA} \cdot B=1 \mathrm{~T}, P_{\text {gas }}=20 \mathrm{~Pa}$.

Since the electron density for $I_{\mathrm{D}}=1 \mathrm{~mA}$ is lower than that for $I_{\mathrm{D}}=3 \mathrm{~mA}$, the sheath electric field formed above the ionic liquid is small for $I_{\mathrm{D}}=1 \mathrm{~mA}$. Therefore, the ion irradiation energy to the ionic liquid is small, and as a result, the ion cannot dissociate the C-F bond of the ionic liquid. However, the C-H bond which has relatively low dissociation energy $(4.29 \mathrm{eV})$ is dissociated by the low-energy ions or neutral nitrogen radicals. The dissociated hydrogen radicals can reduce the Au ions, resulting in the formation of the Au nanoparticles in the plasma irradiation region for $I_{\mathrm{D}}=1 \mathrm{~mA}$.

For $I_{\mathrm{D}}=3 \mathrm{~mA}$, on the other hand, the sheath electric field becomes large in the plasma irradiation region, and then, the high-energy ion irradiation can dissociate the C-F bond of the ionic liquid. Therefore, the Au ions are difficult to be reduced by the oxidation effect of the fluorine radical in the plasma irradiation region. However, in the shadow region of the disk electrode, the $\mathrm{C}-\mathrm{H}$ bond of the ionic liquid is dissociated by the nitrogen radical of the plas$\mathrm{ma}$, and the dissociated hydrogen radical can synthesize the Au nanoparticles. In the center of the shadow region of the disk electrode, the Au nanoparticles are not synthesized because the nitrogen radicals cannot reach to the center of the shadow region.

Based on these results, it is found that the Au nanoparticles are synthesized by the reduction effect via the neutral radical irradiation, and are destroyed by the oxidation effect via the high-energy ion irradiation in the plasma irradiation region. 


\subsection{Size control of ring-shaped nanoparticle structure under inhomogeneous conversing magnetic fields}

For the purpose of size control of the ring-shaped nanoparticle structure, we use the plasma - ionic liquid interface under the inhomogeneous converging magnetic fields (B) to shrink the size of the nanoparticle structure [33].

Figure 12 shows the schematic of a new experimental setup for the discharge plasma in contact with the ionic liquid containing a gold chloride $\left(\mathrm{HAuCl}_{4}\right)$, where the distance between a ring electrode and the ionic liquid on the glass plate is defined as $z_{\mathrm{r}}$. The plasma is generated between a plate cathode electrode and a mesh anode electrode, and is irradiated to the ionic liquid. Here, a mirror ratio $R_{\mathrm{m}}$ is defined as the ratio of the magnetic field strength at the ring electrode position $\left(B_{\mathrm{RG}}\right)$ to that at the ionic liquid substrate position $\left(B_{\mathrm{IL}}\right)$.

(a)

(b)

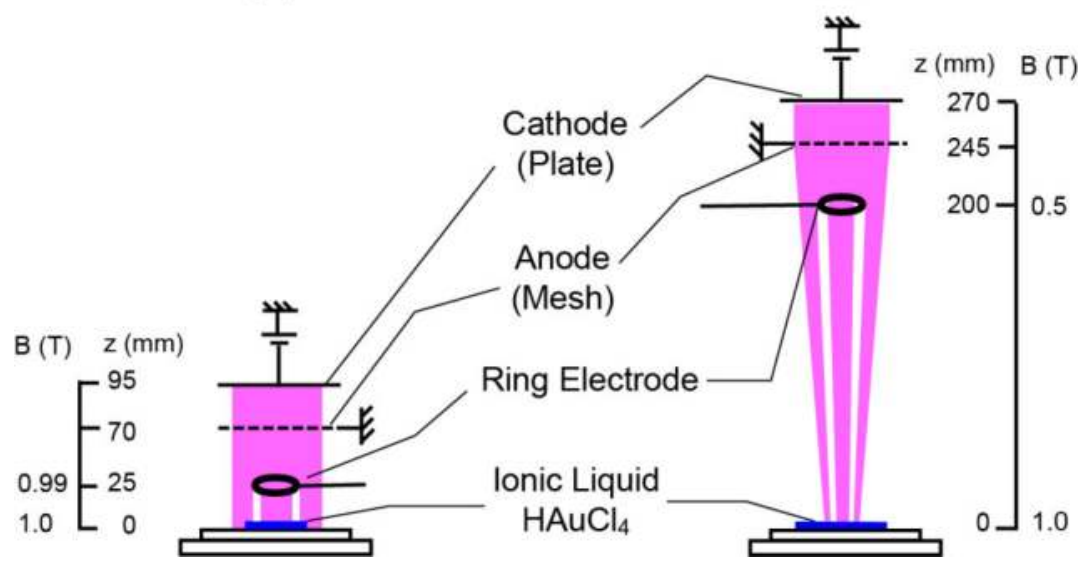

Figure 12. Experimental apparatus for size control synthesis Au nanoparticles under the inhomogeneous converging magnetic fields for mirror ratio (a) $R_{\mathrm{m}} \sim 1$ and (b) $R_{\mathrm{m}}=2$.

Figure 13(a) shows pictures of the Au nanoparticle structure which is formed at the plasma-liquid interface in accordance with the plasma structure for $z_{\mathrm{r}}=25 \mathrm{~mm}$, i.e. $R_{\mathrm{m}} \sim 1$. The $\mathrm{Au}$ nanoparticles are synthesized in the shadow region under the ring electrode with the diameter of about $7 \mathrm{~mm}$, where there are a large amount of neutral radicals and few charged ions. The Au nanoparticles are synthesized by the reduction effect of the radical in the plasma, while the charged ions inhibit the synthesis of the Au nanoparticles by the oxidation effect.

It is possible to control the Au nanoparticle structure by using shrinkage of the plasma diameter under the converging magnetic field. Figures 13(b) and 13(c) show pictures of the Au nanoparticle structure for $z_{\mathrm{r}}=200 \mathrm{~mm}\left(R_{\mathrm{m}}=2\right)$ and $550 \mathrm{~mm}\left(R_{\mathrm{m}}=10\right)$, respectively. 
The diameters of the Au nanoparticle structures are observed to be $5 \mathrm{~mm}$ and $2.2 \mathrm{~mm}$ for $R_{\mathrm{m}}=2$ and $R_{\mathrm{m}}=10$, respectively. Since the diameter of the plasma is proportional to the square root of the mirror ratio, the diameter of the ring shaped nanoparticles is also changed corresponding to the plasma diameter. Therefore, the diameter of the ring-shaped Au nanoparticle structure for $z_{\mathrm{r}}=200 \mathrm{~mm}$ and $550 \mathrm{~mm}$ should be $1 / \sqrt{2}$ and $1 / \sqrt{10}$, respectively. We can easily control the size of the nanoparticle structure by simply change the magnetic field configuration, which is useful for the future nanoparticle nano-electronics devices.
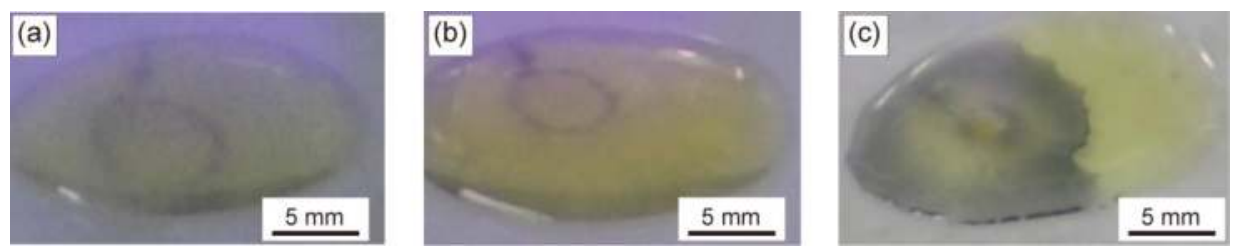

Figure 13. Picture of nanoparticle structures for $(\mathrm{a}) R_{\mathrm{m}}=1$, (b) $R_{\mathrm{m}}=2$, and (c) $R_{\mathrm{m}}=10 . I_{\mathrm{D}}=2 \mathrm{~mA}, P_{\mathrm{gas}}=20 \mathrm{~Pa}, B_{\mathrm{IL}}=1 \mathrm{~T}$.

\section{Conclusion}

A direct current (DC) discharge plasma has stably been generated just above the ionic liquid by applying the DC voltage to an electrode immersed in the ionic liquid. The precise potential structure and the resultant plasma ion or electron irradiation to the ionic liquid are controlled. This ion irradiation is found to be effective for the synthesis of gold nanoparticles in comparison with the conventional electron irradiation system, and the control of the plasma-ion irradiation to the ionic liquid has the possibility of application to the synthesis of the various kinds of size- and yield-controlled nanoparticles.

Furthermore, the periodic and ring shaped Au nanoparticle structures are formed, which correspond to the shape of the strongly-magnetized plasmas generated using the mesh anode electrode or the ring/disk electrode inserted in the plasma.

It is very interesting that the structure of the Au nanoparticles depends on the discharge current, namely, the Au nanoparticles are synthesized from the periphery and absence in the shadow region of the disk electrode for small discharge current, while the Au nanoparticles are synthesized at the boundary of the disk electrode for relatively large discharge current. These phenomena are well explained by the reduction and oxidation effects of the radicals which are generated by the plasma irradiation to the ionic liquid and resultant dissociation of the ionic liquid.

Finally, the size of the Au nanoparticle structure can be controlled by using shrinkage of the plasma diameter under the converging magnetic field, which enables us to freely form the micro- or nano-sized nanoparticle structures. 


\section{Acknowledgements}

The authors thank Prof. K. Tohji, K. Motomiya, T. Miyazaki, and H. Ishida for their technical assistance. We express our gratitude to Dr. K. Baba, Dr. Q. Chen, T. Harada, and T. Okuno for their collaboration. This work was supported by a Grant-in-Aid for Scientific Research from the Ministry of Education, Culture, Sports, Science and Technology, Japan.

\section{Author details}

Toshiro Kaneko, Shohei Takahashi and Rikizo Hatakeyama

Department of Electronic Engineering, Tohoku University, Sendai, Japan

\section{References}

[1] Gubkin J. Electrolytische Metallabscheidung an der freien Oberfläche einer Salzlösung. Annalen der Physik 1887; 268(9), 114-115.

[2] Kanzaki Y., Hirabe M., Matsumoto O. Glow Discharge Electrolysis of Aqueous Sulfuric Acid Solution in Various Atmosphere. Journal of The Electrochemical Society 1986; 133(11), 2267-2270.

[3] Baba K., Kaneko T., Hatakeyama R. Ion Irradiation Effects on Ionic Liquids Interfaced with RF Discharge Plasmas. Applied Physics Letters 2007; 90(20), 201501-1-3.

[4] Kaneko T., Baba K., Hatakeyama R. Static Gas-Liquid Interfacial Direct Current Discharge Plasmas Using Ionic Liquid Cathode. Journal of Applied Physics 2009; 105(10), 103306-1-5.

[5] Bruggeman P., Leys C. Non-Thermal Plasmas in and in Contact with Liquids. Journal of Physics D: Applied Physics 2009; 42(5), 053001-1-28.

[6] Koo I. G., Lee M. S., Shim J. H., Ahn J. H., Lee W. M. Platinum Nanoparticles Prepared by a Plasma-Chemical Reduction Method. Journal of Materials Chemistry 2005; 15(38), 4125-4128.

[7] Hieda J., Saito N., Takai O. Exotic Shapes of Gold Nanoparticles Synthesized Using Plasma in Aqueous Solution. Journal of Vacuum Science and Technology A 2008; 26(4), 854-856.

[8] Richmonds C., Sankaran R. M. Plasma-Liquid Electrochemistry: Rapid Synthesis of Colloidal Metal Nanoparticles by Microplasma Reduction of Aqueous Cations. Applied Physics Letters 2008; 93(13), 131501-1-3. 
[9] Sato S., Mori K., Ariyada O., Atsushi H., Yonezawa T. Synthesis of Nanoparticles of Silver and Platinum by Microwave-Induced Plasma in Liquid. Surface and Coatings Technology 2011; 206(5), 955-958.

[10] Chen L., Iwamoto C., Omurzak E., Takebe S., Okudera H., Yoshiasa A., Sulaimankulova S., Mashimo, T. Synthesis of Zirconium Carbide $(\mathrm{ZrC})$ Nanoparticles Covered with Graphitic "Windows" by Pulsed Plasma in Liquid. RSC Advances 2011; 1(6), 1083-1088.

[11] Seddon K. R. Ionic Liquids: A Taste of the Future. Nature Materials 2003; 2(6), 363-365.

[12] Rogers R. D., Seddon K. R. Ionic Liquids - Solvents of the Future?. Science 2003; 302(5646), 792-793.

[13] Baba K., Kaneko T., Hatakeyama R. Efficient Synthesis of Gold Nanoparticles Using Ion Irradiation in Gas-Liquid Interfacial Plasmas. Applied Physics Express 2009; 2(3), 035006-1-3.

[14] Kaneko T., Baba K., Harada T., Hatakeyama R. Novel Gas-Liquid Interfacial Plasmas for Synthesis of Metal Nanoparticles. Plasma Processes and Polymers 2009; 6(11), 713-718.

[15] Kaneko T., Chen Q., Harada T., Hatakeyama R. Structural and Reactive Kinetics in Gas-Liquid Interfacial Plasmas. Plasma Sources Science and Technology 2011; 20(3), 034014-1-8.

[16] Meiss S. A., Rohnke M., Kienle L., Zein El Abedin S., Endres F., Janek J. Employing Plasmas as Gaseous Electrodes at the Free Surface of Ionic Liquids: Deposition of Nanocrystalline Silver Particles. ChemPhysChem 2007; 8(1), 50-53.

[17] Torimoto T., Okazaki K., Kiyama T., Hirahara K., Tanaka N., Kuwabata S. Sputter Deposition onto Ionic Liquids: Simple and Clean Synthesis of Highly Dispersed U1trafine Metal Nanoparticles. Applied Physics Letters 2006; 89(24), 243117-1-3.

[18] Xie Y. B., Liu C. J. Stability of Ionic Liquids under the Influence of Glow Discharge Plasmas. Plasma Processes and Polymers 2008; 5(3), 239-245.

[19] Kuwabata S., Tsuda T., Torimoto T. Room-Temperature Ionic Liquid. A New Medium for Material Production and Analyses under Vacuum Conditions. The Journal of Physical Chemistry Letters 2010; 1(21), 3177-3188.

[20] Wei Z., Liu C. J. Synthesis of Monodisperse Gold Nanoparticles in Ionic Liquid by Applying Room Temperature Plasma. Materials Letters 2011; 65(2), 353-355.

[21] Kareem T. A., Kaliani A. A. I-V Characteristics and the Synthesis of ZnS Nanoparticles by Glow Discharge at the Metal-Ionic Liquid Interface. Journal of Plasma Physics 2012; 78(2), 189-197. 
[22] Shaw C. P., Fernig D. G., Levy R. Gold Nanoparticles as Advanced Building Blocks for Nanoscale Self-Assembled Systems. Journal of Materials Chemistry 2011; 21(33), 12181-12187.

[23] Huang L., Tu C. C., Lin L. Y. Colloidal Quantum Dot Photodetectors Enhanced by Self-Assembled Plasmonic Nanoparticles. Applied Physics Letters 2011; 98(11), 113110-1-3.

[24] Maye M. M., Nykypanchuk D., van der Lelie D., Gang O. DNA-Regulated Microand Nanoparticle Assembly. Small 2007; 3(10), 1678-1682.

[25] Chen Q., Kaneko T., Hatakeyama R. Rapid Synthesis of Water-Soluble Gold Nanoparticles with Control of Size and Assembly Using Gas-Liquid Interfacial Discharge Plasma. Chemical Physics Letters 2012; 521, 113-117.

[26] Yu J., Rance G. A., Khlobystov A. N. Electrostatic Interactions for Directed Assembly of Nanostructured Materials: Composites of Titanium Dioxide Nanotubes with Gold Nanoparticles. Journal of Materials Chemistry 2009; 19(47), 8928-8935.

[27] Kaneko T., R. Hatakeyama R. Creation of Nanoparticle-Nanotube Conjugates for Life-Science Application Using Gas-Liquid Interfacial Plasmas. Japanese Journal of Applied Physics 2012; in press.

[28] Baba K., Kaneko T., Hatakeyama R., Motomiya K., Tohji K. Synthesis of Monodispersed Nanoparticles Functionalizing Carbon Nanotubes in Plasma-Ionic Liquid Interfacial Fields. Chemical Communications 2010; 46(2), 255-257.

[29] Georgakilas V., Gournis D., Tzitzios V., Pasquato L., Guldie D. M., Prato M. Decorating Carbon Nanotubes with Metal or Semiconductor Nanoparticles. Journal of Materials Chemistry 2007; 17(26), 2679-2694.

[30] Wildgoose G. G., Banks C. E., Compton R. G. Metal Nanoparticles and Related Materials Supported on Carbon Nanotubes: Methods and Applications. Small 2006; 2(2), 182-193.

[31] Ye X., Lin Y., Wang C., Engelhard M. H., Wang Y., Wai C. M. Supercritical Fluid Synthesis and Characterization of Catalytic Metal Nanoparticles on Carbon Nanotubes. Journal of Materials Chemistry 2004; 14(5), 908-913.

[32] Han L., Wu W., Kirk F. L., Luo J., Maye M. M., Kariuki N. N., Lin Y., Wang C., Zhong C.-J. A Direct Route toward Assembly of Nanoparticle-Carbon Nanotube Composite Materials. Langmuir 2004; 20(14), 6019-6025.

[33] Kaneko T., Takahashi S., Hatakeyama R. Control of Nanoparticle Synthesis Using Physical and Chemical Dynamics of Gas-Liquid Interfacial Non-Equilibrium Plasmas. Plasma Physics and Controlled Fusion 2012; in press.

[34] Krenn J. R. Nanoparticle Waveguides: Watching Energy Transfer. Nature Materials 2003; 2(4), 210-211. 
